AIAA-21000-0052

\title{
HARDWARE AND SOFTWARE DEVELOPMENTS FOR THE ACCURATE TIME-LINKED DATA ACQUISITION SYSTEM ${ }^{*}$
}

\author{
Dale E. Berg ${ }^{\dagger}$, Mark A. Rumsey ${ }^{\ddagger}$ \\ Wind Energy Technology Department \\ Sandia National Laboratories \\ Albuquerque, New Mexico \\ Jose R. Zayas \\ General Technology Corporation \\ Albuquerque, New Mexico
}

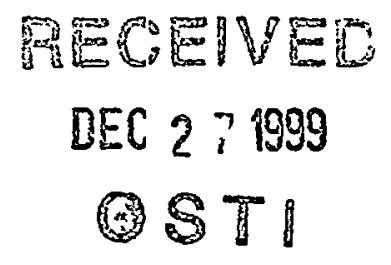

\begin{abstract}
Wind-energy researchers at Sandia National Laboratories have developed a new, light-weight, modular data acquisition system capable of acquiring long-term, continuous, multi-channel time-series data from operating wind-turbines. New hardware features have been added to this system to make it more flexible and permit programming via telemetry. User-friendly Windows-based software has been developed for programming the hardware and acquiring, storing, analyzing, and archiving the data. This paper briefly reviews the major components of the system, summarizes the recent hardware enhancements and operating experiences, and discusses the features and capabilities of the software programs that have been developed.
\end{abstract}

\section{INTRODUCTION}

Personnel in the Wind Energy Technology Department at Sandia National Laboratories, a part of the National Wind Technology Center (NWTC), began the development of hardware and software for a new, stateof-the-art wind turbine data acquisition system in late 1997. The primary design goal of the new system was that it be capable of acquiring long-term, continuous, multi-channel time series data from operating wind turbines. We have developed the Accurate, TimeLinked Data Acquisition System (ATLAS) hardware and software to achieve this goal.

\footnotetext{
- This work was supported by the U.S. Department of Energy under contracts DE-AC04-94AL85000 and DEAC36-83CH10093.

+ Principal Member of Technical Staff, Associate Fellow of AIAA.

"Senior Member of Technical Staff.

${ }^{\S}$ This paper is declared a work of the U.S. Government and is not subject to copyright protection in the United States.
}

A complete wind-turbine data acquisition conf guration typically contains several data acquisition subsystems (DAS). Normally, a system configuration urilizes at least one rotor-based DAS unit (RBU), at least one ground-based DAS unit (GBU) and one ground-based computer unit (GBCU), as illustrated in Figure 1. The $\mathrm{RBU}$ is mounted on the rotor, rotating with and in close proximity to the blade- and main shaft-mounted strain gauges and other sensors. The GBUs are any' and all data acquisition units that don't mount on the rotor-a GBU could be mounted in the turbine nacelle, on the turbine tower, or on the meteorological tower. All acquisition units are located close to the sensors from which they are acquiring data in order to minimize contamination of the data by electrical noise picked up by long signal wires. The ground-based control unit (GBCU) is the heart and brains of the system. The GBCU queries the user to determine the configuration of each DAS (the channels to sample, the channel gains, offsets and filters, and the rate at which cata is to be sampled); programs each DAS to perform the acquisition; performs calibrations; retrieves and collates the data acquired by each DAS; and displays and stores that data to disk. The smart data acquisition system (SDAS) retrieves that data from disk, post processes it, and archives it.

ATLAS utilizes commercially available data acquisition, telemetry, and global positioning system (GPS) receiver hardware, together with a custom-built programmable logic device subsystem (PLDS), to acquire long-term time series data from multijle data acquisition units. The PLDS utilizes the GPS receivers to maintain precise time synchronization between the various DAS units, forcing data acquisition on all units at exactly the same time over the entire duration of a test. An ATLAS prototype, with a mixture of Windows- and DOS-based software, has been installed on the Atlantic Orient Corporation 15/50 wind turbine at the U.S. Department of Agriculture (USDA) Agricultural Research Service (ARS) site at Bushland 


\section{DISCLAIMER}

This report was prepared as an account of work sponsored by an agency of the United States Government. Neither the United States Government nor any agency thereof, nor any of their employees, make any warranty, express or implied, or assumes any legal liability or responsibility for the accuracy, completeness, or usefulness of any information, apparatus, product, or process disclosed, or represents that its use would not infringe privately owned rights. Reference herein to any specific commercial product, process, or service by trade name, trademark, manufacturer, or otherwise does not necessarily constitute or imply its endorsement, recommendation, or favoring by the United States Government or any agency thereof. The views and opinions of authors expressed herein do not necessarily state or reflect those of the United States Government or any agency thereof. 


\section{DISCLAIMER}

Portions of this document may be illegible in electronic image products. Images are produced from the best available original document. 
(near Amarillo), Texas. Prominent features of the ATLAS include:

- Provides a complete integrated package using offthe-shelf components, thereby minimizing custom electronics design, system integration, and software support and development functions.

- Provides flexible, expandable hardware that can be integrated with common experiment-control software to provide necessary quick-look data display, processing, and analysis capabilities.

- Decreases the time and effort required to instrument a field experiment, perform calibrations, verify data validity, collect data, and do preliminary data analysis, improving the end-toend accuracy of data collected in field experiments.

- Provides a versatile system capable of making a wide variety of reliable measurements in rugged environments using equipment of unobtrusive size and minimal weight.

- Provides stand-alone operation by incorporating telemetry and battery packs that can be recharged on site.

- Provides the capability to acquire and save data to disk at rates up to 12,500 samples per second, enabling the recording of contiguous long-period time records, so unexpected transients will not be missed, and available testing time is not wasted.

- Provides an easy way to get experimental data into a standard PC data file format, thus enabling users of varied computer skill and experience levels to use any standard PC-based software products to perform post-processing analyses and displays.

Additional information on the ATLAS hardware development may be found in references 1-4.

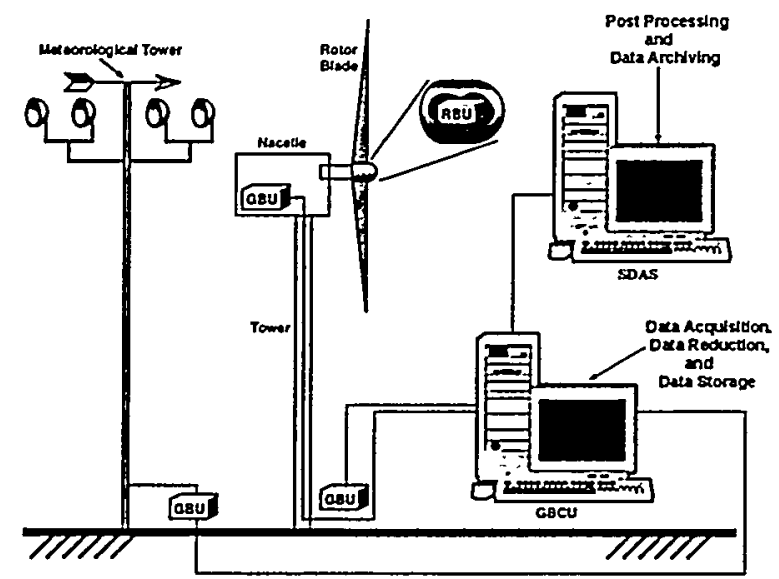

Figure 1. Schematic of Typical Wind Turbine Data Acquisition System
The ATLAS hardware is only part of the system. In order to tailor the system for wind turbine use and make it user-friendly and flexible, powerful, user-iriteractive software is required. As mentioned in the earlier references, we have chosen to develop the soitware in the National Instruments LabVIEW ${ }^{\circ}$ for W/indows ${ }^{5}$ environment, both to facilitate the development of a single data acquisition software system (the ADAS II software) for this and other hardware system!s, and to utilize LabVIEW's power to develop a roluust and friendly user-interface. Some hardware deve opments have been accomplished during the months since the last paper on this system was published ${ }^{4}$, but most of the development has been on the software side, with the development of the ATLAS program to configure the hardware, the ADAS II program to acquire and store the data, and the SDAS program to post process, analyze, and archive the data. All of these programs are LabVIEW executables and require no software license or additional software to run.

\section{RECENT HARDWARE DEVELOPMEIYTS}

Several additional hardware features have been added to the PLDS to yield new capabilities since the prototype was deployed on the AOC turbine diring the summer of 1998.

The GPS receiver is no longer mounted on the PLDS card as a daughter card. Instead, the receiver is now connected to the PLDS via a 9-wire cable and is mounted in a small, sealed enclosure immediately adjacent to the GPS antenna. The new configuration is pictured in Figure 2. This reduces the siz: of the PLDS, so that it only requires one slot in the DAS mainframe, freeing up room for additioral data acquisition modules. It also enables the user to mount the antenna for GBUs several hundred feet away from the PLDS, with no need to be concerned about attenuation of the GPS antenna signal, as was the case with the earlier configuration. The RBU anterina must still be mounted on the rotor axis of rotation, so it will still be close to the PLDS.

An analog-to-digital conversion chip has been added to the PLDS board to facilitate measurement of temperatures, currents, and voltages. This enables the user to track the health and status of the card and the new battery power supply. We are now utilizing lowerpower GPS receivers (dropping the power consumption from 1.1 watt to 0.2 watts) and have implemented a "sleep" mode to further conserve power when data are not being acquired. This would normally be used only in the RBU, where a battery pack is used for power. In this mode, we switch off the power to the DAS mainframe, all the DAS modules, the modems, and 
selected components of the PLDS module for 50 seconds of every minute. The programmable logic device (PLD) on the PLDS module remains alive, and will turn on the telemetry components for the first 10 seconds of each minute to check for communications from the user. The RBU remains in this mode until a wake up call is received. These features result in a dramatic reduction in power consumption. For example, the ATLAS RBU on the AOC turbine, which provides excitation power to and acquires data from 15 strain gauges, now consumes about 32.5 watts of power whenever it is acquiring data or not. The new version of the PLDS enables us to place the unit into sleep mode when not actually acquiring data. This drops the power consumption to about 2 watts. Whenever we are ready to take data, we wake the unit up and within five seconds it is consuming 32.5 watts and acquiring data.

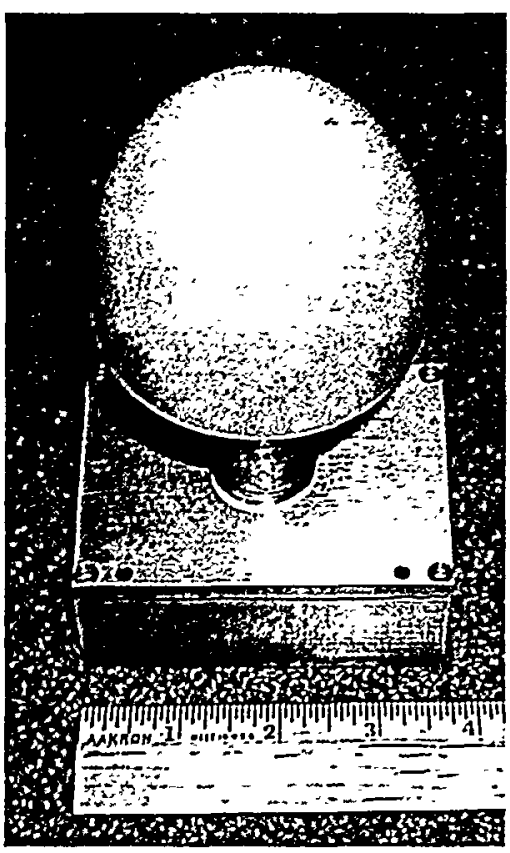

Figure 2. GPS Antenna and Receiver Enclosure

The PLD logic program has been enhanced to latch the GPS time (accurate to one microsecond) when the analog and strain-gauge data are actually acquired. Including this time in the data stream gives the user a powerful tool for determining if and when communication dropouts occur. In addition, the user can now direct the PLDS to

- transmit the GPS data to the GBCU

- connect the full duplex communications between the GBCU and the DAS (for remote programming of the DAS)

- transmit the DAS data via serial link to the GBCU
- transmit the unique PLDS identification cade to the GBCU

- reset the PLDS

- enter or exit the sleep mode

- turn DAS (12-volt) power on or off

- turn GPS (5-volt) power on or off

- turn the PLDS-generated external pulse train generation on or off.

A prototype battery system permits the installation of an RBU on a rotor that has no electrical power. The 15-pound unit consists of nickel/metal hydride cells and has a nominal rating of $48 \mathrm{amp}$ hours at 12 VDC. The nickel/metal hydride cells have twice the energy density of the more common nickel/cadmium cells and do not suffer from the memory effect (a loss of ability to accept charge and an increase in internal resistance) that affects those cells. Nickel/metal hydride cells can be recharged hundreds of times with little or no degradation and do not require a complex charging unit, unlike the higher energy-density but less robust lithiumion cells. With this battery pack and the sleep mode of the new PLDS card, we could run the AOC rotor unit for a full week (168 hours), assuming we acizuire no more than 8 hours of data (the unit is in sleep rnode for 160 hours or more). The system will provide power for about 18 hours of continuous data acquisition. 'We have not yet had the opportunity to gain any field experience with this unit.

\section{ATLAS SOFTWARE PROGRAM}

The computer requirements necessary to run the ATLAS software package are

- Pentium $200 \mathrm{MHz}$ or faster

- $32 \mathrm{Mb}$ of RAM (64Mb recommended)

- $20 \mathrm{Mb}$ disk space

- $800 \times 600$ minimal resolution with small fonts (1024 X 768 recommended)

- Windows 95 or 98

The ACRA Control ${ }^{6}$ KAM 500 (also sold in the US as Nicolet MicroPro) that we use for the DAS unit was supplied with a rudimentary 16-bit DOS-based programming/data acquisition software. While this software was adequate for initial system development, it was apparent that we needed to develop software that would operate under and take full advantage of the 32bit Windows environment. The new ATLAS software (written in LabVIEW) allows the user to specify the hardware configuration of all the DAS units, to specify the setup (actual hardware, gain, filter setting, etc.) for each channel, and to actually program the DAS units to acquire the desired data. 
Figure 3 shows the front panel of the ATLAS software. From here, the program takes the user through a series of steps to configure and program the ATLAS hardware. The first thing the user must do is configure the hardware by selecting the "Configure Hardware" button from the front panel. This brings up the window shown in Figure 4, where he can select the size of the DAS mainframe (the number of data-acquisition module slots) and the different data acquisition modules that are inserted. If the hardware configuration contains one or more slave units, the user can also specify the mainframe size and modules inserted in those units at this time. Once the hardware configuration is complete, the user clicks the "OK" button and is returned to the front panel.

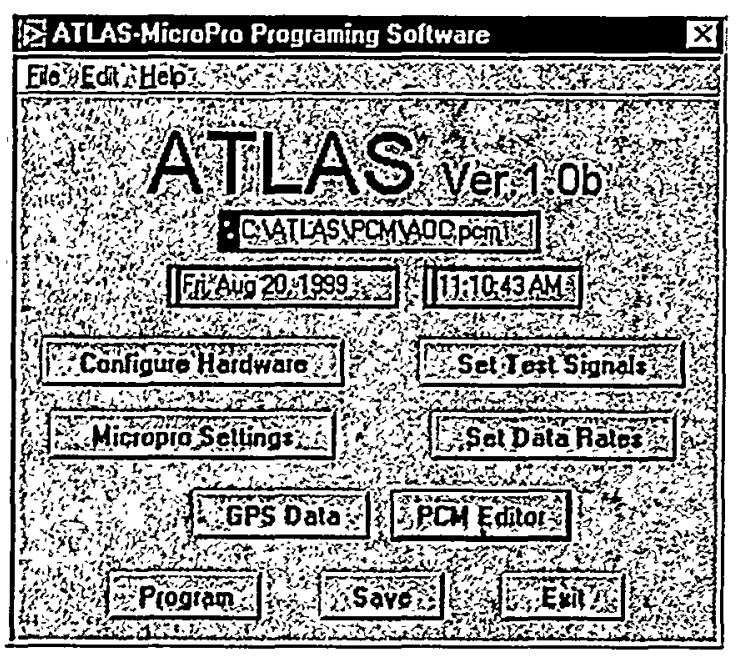

Figure 3. ATLAS Software Front Panel.

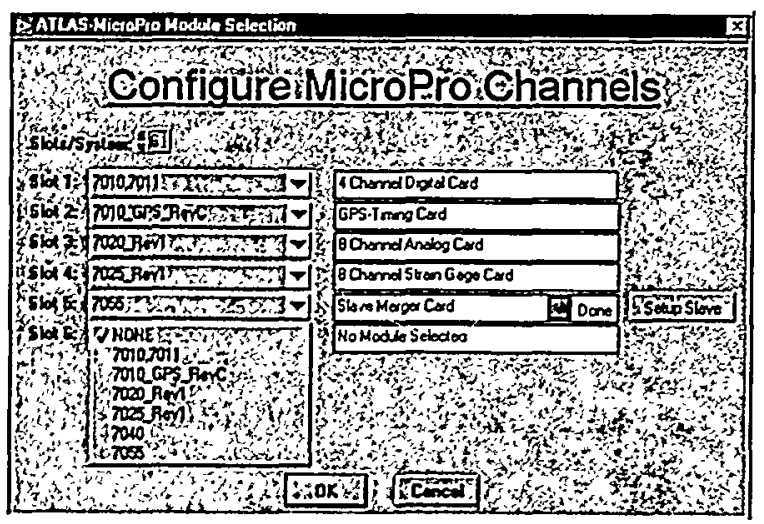

Figure 4. Configure Hardware Window. Notice the Setup Slave button on the right.

The communications protocol to be used by the various DAS units is the next parameter to be specified using the "MicroPro Settings" button, which brings up the window shown in Figure 5. Here the data word size, the sync word, and the type of data encodin; (NRZ-L or Bi-phase) must be specified. These settings apply to all DAS units in the ATLAS. Whether the mode of data communication is TTL or RS-422 must also be specified, and this can vary from DAS to DAS. RS-422 can be utilized to transfer data over distances: of up to one kilometer, while TTL can be utilized if the transfer distance does not exceed about 20 feet. TTL must be utilized if telemetry is being used. The user also can enter a description (255 characters or less) of the data acquisition configuration. When these settings are complete, the user clicks the "OK" button and is returned to the front panel.

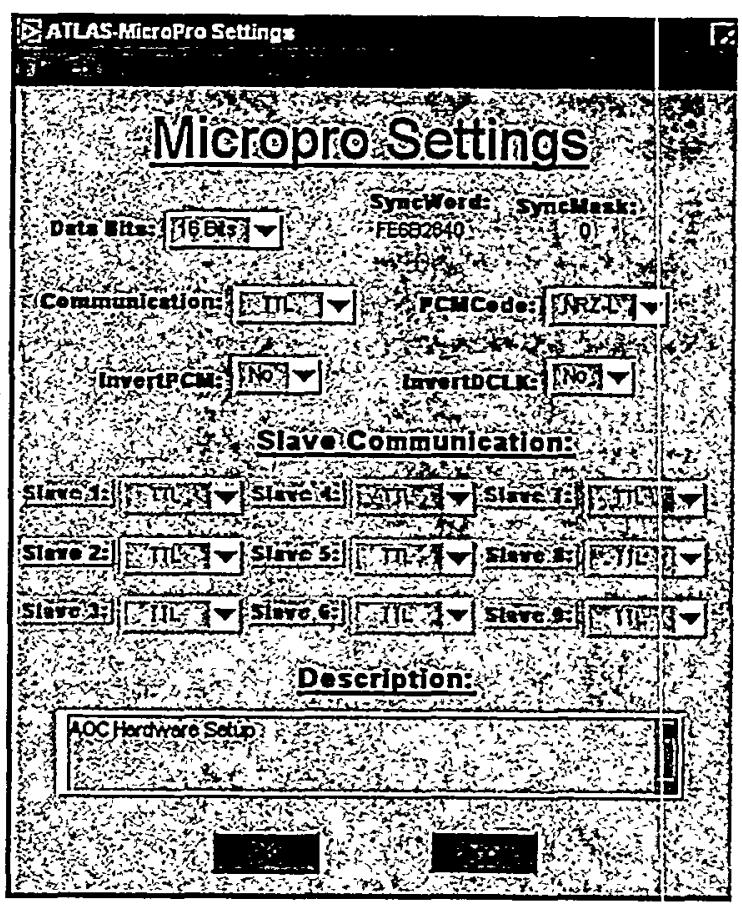

Figure 5. MicroPro Settings Window

The next step defines the data acquisition signals, done with the "Set Test Signals" button, which brings up the window shown in Figure 6. The user selects a module and creates a data channel with the specific information required for that channel. This information includes channel name, filter settings, excitation voltage, amplifier gain, etc. At any time in this process, the user can view the signals that have been definec. in the "Review" window and edit or delete any existing signals. After all of the signals have been defined, the user clicks the "OK" button and again is returned to the front panel. 
The last step is to select the sampling rate (all channels are sampled at the same rate), done with the "Set Data Rates" button, which brings up the window shown in Figure 7. After the desired data rate is specified, the software computes the closest data rate that can actually be realized, given the number of bits being acquired for each sample and the internal $8 \mathrm{MHz}$ clock. In general, this rate is slightly higher than the user-specified one. In the example in Figure 7, the user has requested a 30$\mathrm{Hz}$ sample rate. The actual rate, based on the $8 \mathrm{MHz}$ clock and 48 channels of 16-bit words (12 bits of data and 4 bits of blank fill), is $30.02 \mathrm{~Hz}$. However, since the data acquisition signal is synchronized with GPS time every second, the result is exactly 30 samples each second. Clicking on the "OK" button will again return the user to the front panel.

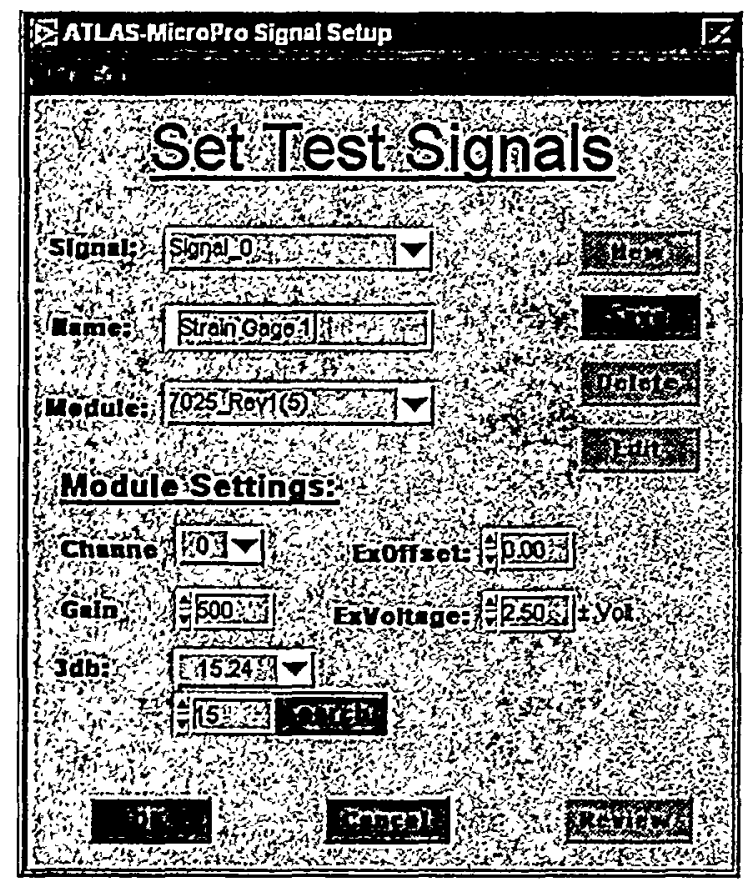

Figure 6. Set Test Signals Window. Notice the Review button on the bottom right.

At this time, the user should save all of the input information with the "Save" button. This creates an ASCII "PCM" file that contains all of the information gathered from the user and necessary to program the hardware. The "PCM Editor" button in the front panel launches Notepad+ (a text editor) to allow the user to view this file. Expert users may also modify the file manually. After the PCM file is saved, the hardware is programmed with the "Program" button. If the ATLAS system contains only one DAS unit, ATJ_AS will immediately start to program the hardware over the RS232 link. If the setup contains slave units, ATLAS can program the entire system sequentially or just individual DAS units as specified by the user. Programming DAS units individually can be useful if a configuration modification to only one unit in the system is made.

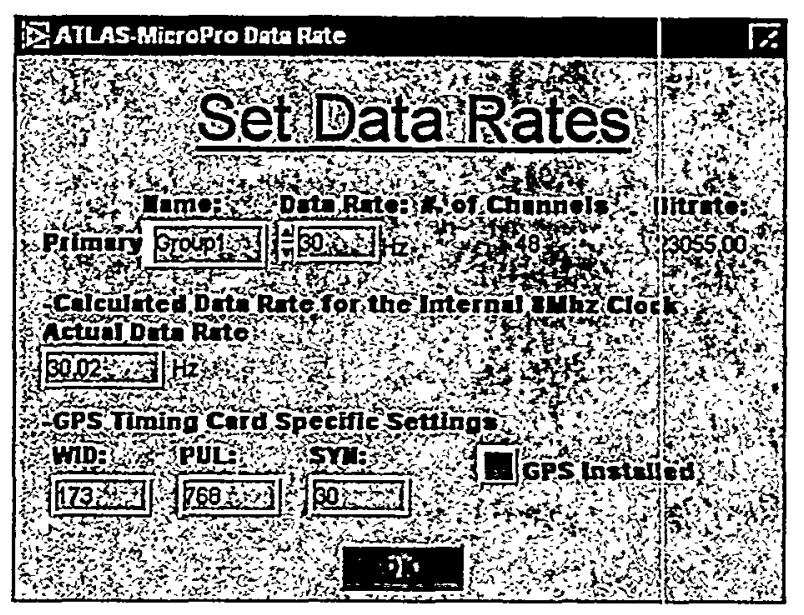

Figure 7. Set Data Rate Window

The program versatility and simplicity allows the user to program an entire data acquisition system in a short amount of time.

Another key feature of the ATLAS software is the ability to monitor and/or control the GPS module, which is only activated if the user has selected the "7010_GPS RevC" module in the "Configure Hardware" window. In this case, the DAS unit in which the PLDS module is located is actually controlled by the signals generated by the PLDS, utilizing the values calculated from user input in "Set Data Rate" window. The "GPS Data" button on the Front Panel window then permits the user to monitor GPS data and to switch the PLDS commurications mode from the GPS Communications window (Figure 8). From this window the user can command the PLDS communication lines to perform one of several possible actions:

- transmit the incoming GPS data to the ground computer

- program the DAS hardware

- transmit the DAS-acquired data to the GBCU serially 
- return the unique PLDS logic software ID information

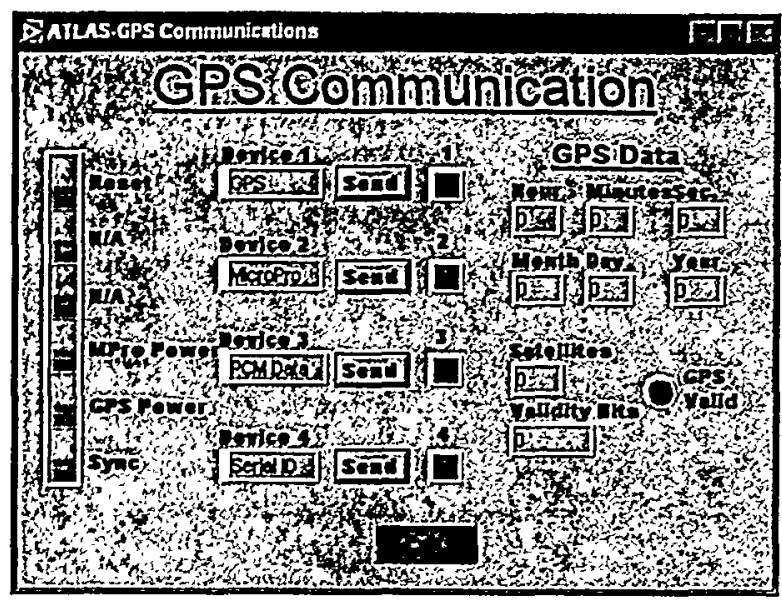

Figure 8. GPS Communication Window

GPS data transmitted to the GBCU is displayed in the GPS Communication window. The "GPS Valid" button in the lower right of the window will turn from red to green when the GPS receiver is actually locked to GPS time. Once this occurs, the DAS unit is ready for data acquisition. This condition must be true for all DAS units in the ATLAS before data acquisition takes place.

\section{ADAS II SOFTWARE PROGRAM}

ADAS II is a modified version of the Advanced Data Acquisition System (ADAS) software developed by NREL for acquiring data from the ADAS hardware. It has been enhanced to acquire and store continuous time-series data from the ATLAS hardware.

This software will run on a computer with the following minimum capabilities:

- Pentium 200MHz or faster

- $\quad 64 \mathrm{Mb}$ of RAM (128Mb recommended)

- $25 \mathrm{Mb}$ disk space (plus necessary disk space for data storage)

- $1024 \times 768$ minimal resolution

- Windows 95 or 98

The Front Panel of the ADAS II software is shown in Figure 9.

ADAS II accesses the PCM file that was created by the ATLAS software and decodes that file to acquire the information it needs to configure itself to actually acquire data from the ATLAS hardware.

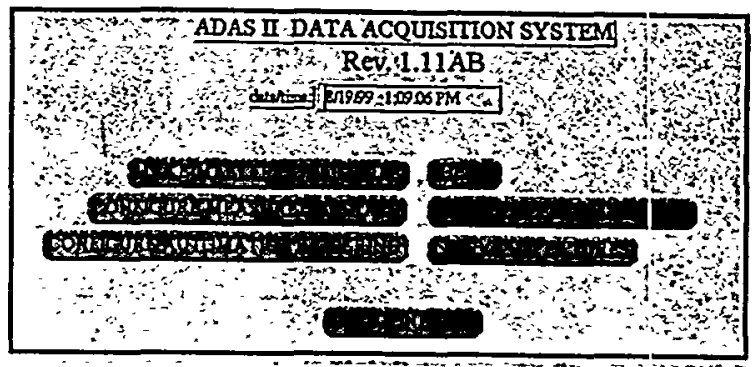

Figure 9. ADAS II Software Front Panel.

The ADAS II Channel Configuration window is shown in Figure 10.

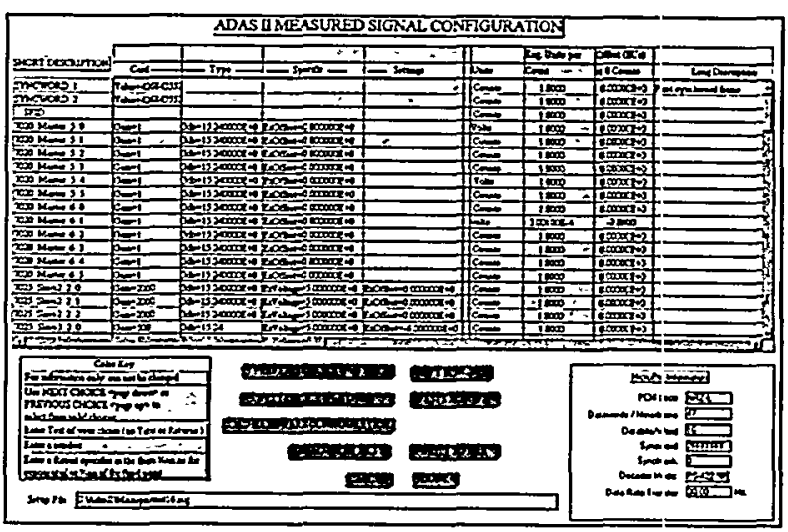

Figure 10. ADAS II Channel Configuration Window

Once the program has been configured, the user must specify the location and the time duration of the data files to be taken, and then the software is ready to acquire continuous data. All saved data files are stored with a header file, which contains all of the Jertinent information that belongs to that specific data file. The data and header files can then be read by many software packages for post processing (NREL prograrns GPP, Crunch, and Genstats, as well as the new SDAS). The "Collect Data" window, shown in Figure 11, is displayed during actual data acquisition. Within this window, the user also has the capability to view the data in pseudo real time in any of several different chart formats.

Other important features of the ADAS II software are the triggering routine and the logbook. The triggering routine allows the user to specify specific quotas or conditions to their data. In other words, if the data does not meet the specific requirements, the data are not saved. For example: If the triggering condition.s are set to take data when the wind speed is greater than $6 \mathrm{~m} / \mathrm{s}$, data will not be saved unless the wind speed exceeds $6 \mathrm{~m} / \mathrm{s}$. 
The logbook feature maintains a record of any modifications, errors, or events that occur while the program is running. The user may view this file at any time, which can be very useful, especially if the program is taking data unattended.

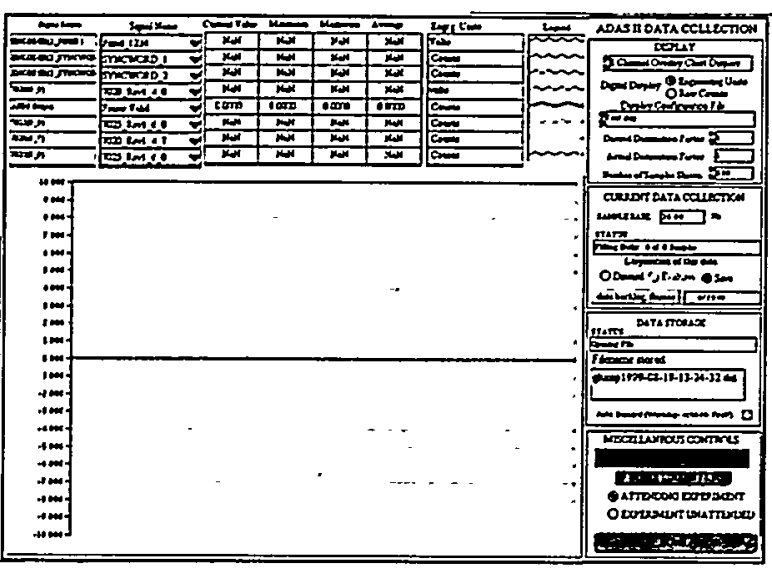

Figure 11. ADAS II Collect Data Window

\section{SDAS SOFTWARE PROGRAM}

\section{SDAS Overview}

A new program called the Smart Data Acquisition System (SDAS) has been written to help researchers process the large amounts of data that are typically acquired in performing wind energy technology research. Data acquired and stored with the ADAS II software can be read directly into SDAS, in effect enhancing the capabilities of the ADAS II program. The SDAS program is intended to complement other existing NREL analysis programs, such as GPP, Genstats and Crunch, which can also read ADAS II time-series files.

SDAS is an interactive stand-alone LabVIEW program. It is a unique data acquisition program; instead of acquiring data from hardware, as is normally done, SDAS acquires data created (or acquired) by other programs, usually, but not necessarily the ADAS II data acquisition program. One of the concepts that is built into SDAS is the ability of the program to know what data have been taken; in a way, SDAS maintains a global picture of what data has been acquired. SDAS is designed to receive a continuous stream of time-series files either from the ADAS II computer program in pseudo-real-time (typically, but not necessarily, every 10 minutes), or off-line from archived time-series files. SDAS can calculate and store an extensive set of userselected statistics for every time-series file. All statistics are stored in a central database that can be accessed later by the user. SDAS will allow a user to interactively interrogate the database or any time-series file that was previously processed by SDAS. This gives the user the ability to perform a large number of tasks including looking at statistics,; looking for trends, finding extrema, looking for particular events,; performing Rainflow analysis, plotting channels or statistics against each another, investigating point-bypoint details in time-series files, determining file commonality (redundant time-series file:s), and dissecting, concatenating, and archiving time-series files.

\section{$\underline{\text { SDAS Computer Requirements }}$}

We knew up front that SDAS could possibly be required to handle large data files, thus requiring it to have large system memory requirements. SIJAS was written to optimize computer memory wherever possible. SDAS is also graphics intensive. It: will run on a computer with the following ininimum capabilities:

- Pentium $200 \mathrm{MHz}$ or faster

- $64 \mathrm{Mb}$ of RAM (128Mb recommended)

- $25 \mathrm{Mb}$ disk space (plus necessary disk space for data storage)

- $1024 \times 768$ minimal resolution with small fonts

- Windows NT

Only one time-series file at a time is read into the SDAS memory during an SDAS acquisition, and up to four time-series files can be read in at a time during post-processing. The only potentially large files that SDAS creates are the SDAS databases, which start out at 74 kilobytes each and grow at a rate of 2 ivilobytes for every time series file acquired and processed.

\section{SDAS Status Window}

The main window in SDAS, called the SDAS Status window, is shown below in Figure 12. The dynamic settings of SDAS, as they are set and changed, are displayed in this window. All of the functions invailable in SDAS can be reached from this window through the (File, Acquisition, Post Processing, View, Utilities, Options, Help) menu bar. As long as SDAS is running, this window will always be on the desktop.

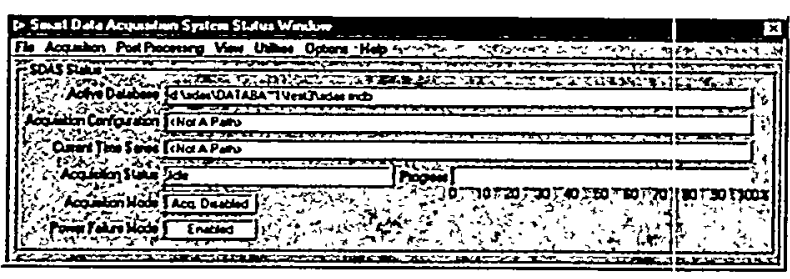

Figure 12. SDAS Status Window. 


\section{SDAS Statistics}

SDAS can calculate a large number of built-in userselected statistics. Along with the minimum and maximum, the four moments (mean, standard deviation, skewness and kurtosis) can be calculated for each data channel. Also, up to thirteen special statistics, called cross-channel statistics, can be calculated. A crosschannel statistic is calculated from more than one data channel, and such statistics are routinely calculated in wind energy research. The list of cross- channel statistics currently available within SDAS includes capacity factor, horizontal sonic direction, horizontal sonic velocity, Obukhov length, Reynolds stress, Richardson number, total sonic velocity, vertical sonic velocity, wind direction, wind shear, yaw error, yaw rate, and turbulence intensity.

\section{SDAS Acquisition Configuration}

Before any data can be acquired, SDAS has to know what statistics are to be calculated and stored in the database for each input channel. The Statistical Analysis Configuration window, shown in Figure 13, allows the user to interactively point to a channel/statistic field intersection and select or deselect the statistics to be calculated and stored for any raw (data) or calculated channel. An $\mathrm{X}$ indicates the statistic will be calculated and stored; the absence of an $\mathrm{X}$ indicates the statistic is not calculated or stored. The vertical list of channels in this window (except the yaw error) is read in from a time-series header file, or a previously saved Statistical Analysis Configuration file.

The Cross Channel Statistics are selected or deselected in the same manner as the simple statistics described above. As can be seen in Figure 14, the yaw error is the only cross-channel statistic selected.

For example, for the yaw error statistic to be calculated, SDAS needs to map two variables to two data channels, done in the SDAS Cross Channel Statistics Configuration window shown in Figure 14. This window is reached by clicking on the button labeled "Edit Cross Channel Statistics" along the bottom of the Statistical Analysis Configuration window. Notice the yaw error variable "wind direction (degrees)" has not been assigned to a data channel yet, but the yaw error variable "yaw angle (degrees)" is about to be assigned to the data channel "rotor yaw angle (degrees)" from the pull-down menu that lists all the data channels. After the yaw error statistic has been configured, the yaw error will now appear in the Statistical Analysis Configuration window. See the bottom entry in Figure
13. At this point, any simple statistics can be selected/deselected for the yaw error statistic.

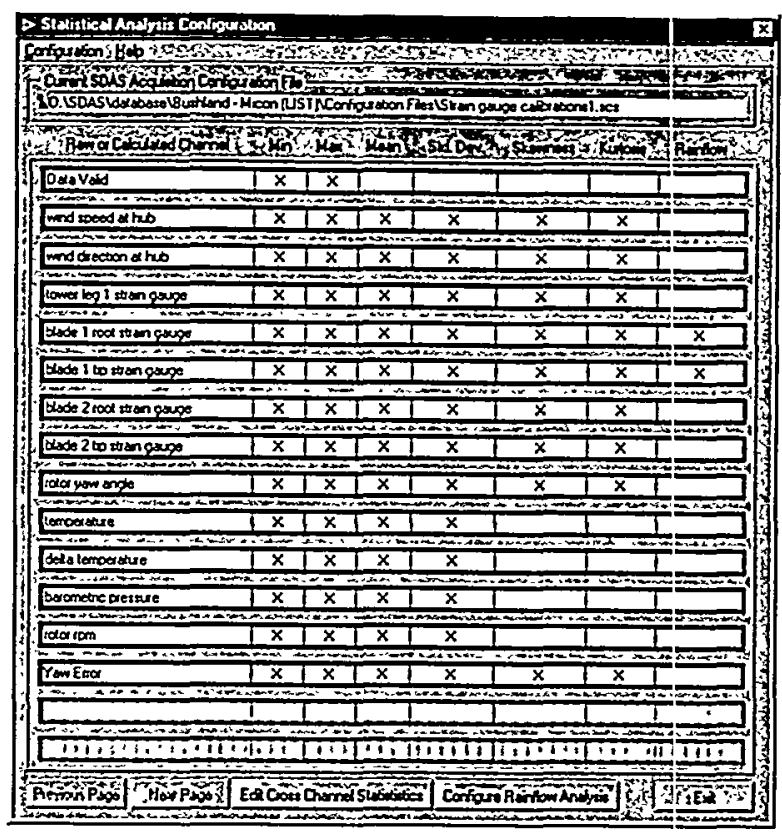

Figure 13. Statistical Analysis Configuration Window. This allows the user to deline the statistics to be calculated for each data and calculated channel.

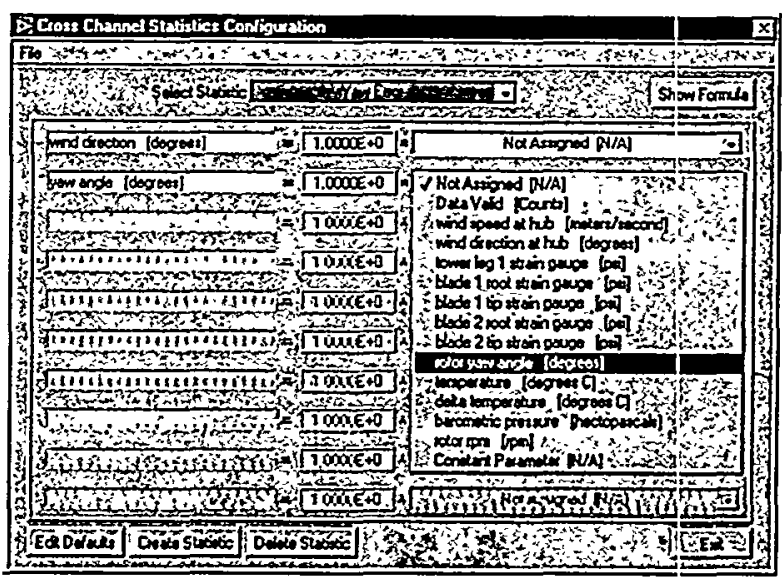

Figure 14. Cross Channel Statistics Configuration Window. The yaw angle variable is about to be mapped to the rotor yaw angle clata channel.

Notice also in Figure 13 that two boxes in the Rainflow column have been selected (with an $X$ in the last column) for the "blade 1 root strain gauge" and "blade 1 tip strain gauge" channels. This means two data channels have been selected for Rainflow analysis, and 
must be configured. The Rainflow configuration window appears in Figure 15. The fields in this window have been user-configured in the same way as described above.

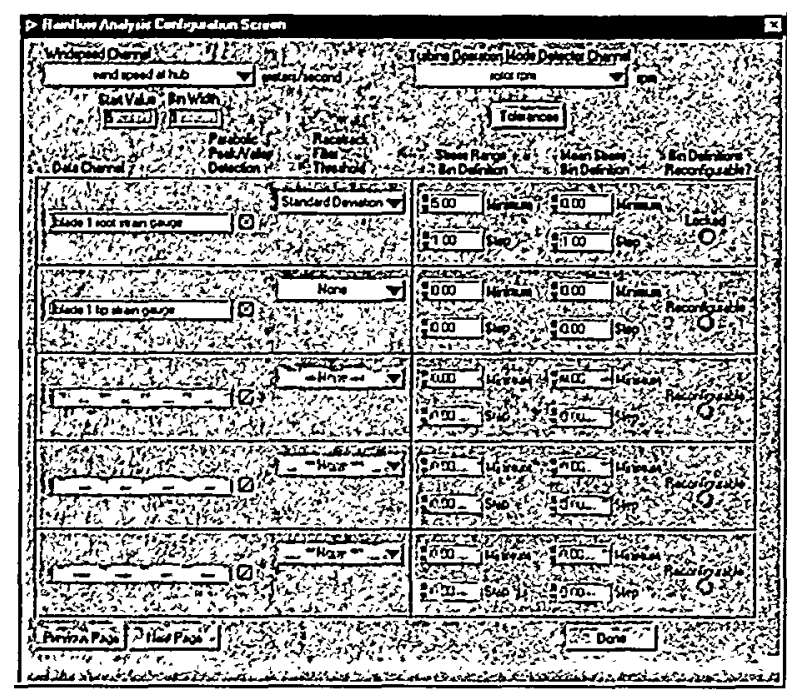

Figure 15. Rainflow Analysis Configuration Window. The user-defined parameters for a rainflow analysis for two data channels have already been set in the Statistical Analysis Configuration window.

After the user has configured the SDAS acquisition by selecting and mapping the statistics, SDAS is ready to start acquiring time-series data. The time-series files can be acquired from a user-designated location on the local computer or over a network. The time-series files could be located in the computer folder where the ADAS II software is storing current time-series files, in another computer folder, or on a CD-R or a floppy disk.

\section{SDAS Database}

In SDAS, a database is the cumulative collection of information obtained by a sequence of acquisitions of time-series data. Each database is a knowledge base of information about a specific grouping of data. For instance, if you have processed data from both the Bergey 850 and the Zond Z-56 wind turbines, you'd probably want to separate that data into at least two groups, one for each turbine. SDAS gives the user the ability to create and maintain any number of databases. However, only one SDAS database can be open at any given time while SDAS is running.

The SDAS database is based on the Microsoft Access database format, and thus can be read by Access. SDAS communicates with the database through the
SQL and Microsoft Open Database Connectivity (ODBC) driver interfaces. However, all of this is transparent to the user. There are no database query commands to remember, and Microsoft Access does not need to be installed on the computer in order to run SDAS.

\section{SDAS Post-processing}

SDAS has several post-processing capabilities. Four different kinds of XY-plots can be displayed, each with one to four channels per plot, and they can be interactively manipulated. They can be Statistic versus Time, Channel versus Time, Statistic versus Statistic and Channel versus Channel. An example of a Channel versus Channel plot, with four channels displayed, is shown in Figure 16. The arrays generated by the Rainflow analysis are plotted using LabVIEW intensity plots.

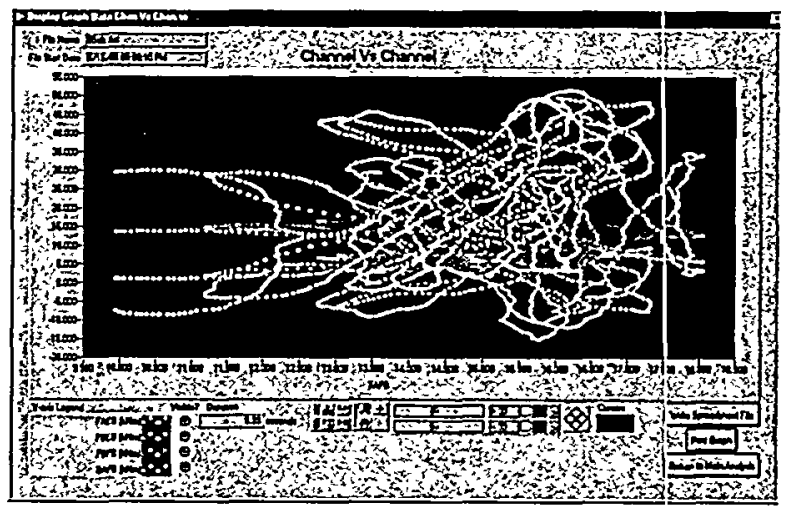

Figure 16. Channel versus Channel Plot. Four strain gauge channels are plotted against a. fifth strain gage channel.

All the powerful LabVIEW graphics functions are available to modify the view of any of the plots (zoom, panning, cursor measurement, auto-scaling, etc.). A Microsoft Excel spreadsheet can be generated from the windowed data in any plot.

Conditions filtering is the name we have given a powerful capability which is similar to the triggering capability of the ADAS II program. The user can set criteria or limitations on the data displayed, in effect filtering information from the database. The criteria are user selectable and are based on a logical combination of any SDAS calculated statistic from any channel and the time the file was created.

A number of SDAS file-manipulation utilities are also available to the user. For example, the user can dissect, 
concatenate, and edit time-series file, generating new time-series files. There is also a powerful dataarchiving function, which permits the user to compress or zip (if so desired) time-series files and write them to CD-R.

\section{SDAS and Power Failures}

Because SDAS may be operated unattended for long periods of time, the program was written with the capability to gracefully handle utility power failures that could affect the integrity of the data acquisition. The ADAS and SDAS computers and ATLAS data acquisition hardware are all on an uninterruptible power supply (UPS). If a power failure occurs, the software that controls the UPS generates a text file in a userdefined folder on one of the computers. If SDAS detects the presence of this text file in the specified folder, SDAS will finish all calculations on the current time-series file, store all calculations in the database, record all the user-defined SDAS settings, and gracefully terminate itself before the SDAS computer is shut down by the UPS. (Currently, the ADAS II software and ADAS computer do not have this powerfailure shutdown capability.) When the utility power recovers, the SDAS computer is powered on by the UPS, self boots, and SDAS is restarted. SDAS will reverse the shutdown process, reestablishing its acquisition configuration, and resume time-series file acquisitions, performing statistical calculations, and storing results in the database, as if nothing had happened.

\section{OPERATING EXPERIENCE}

Two severe lightning strikes on the $A O C$ prevented us from acquiring much data from our prototype installation, even though we installed it over a year ago. The first strike damaged the ATLAS extensively. We added lightning protection to protect most data lines, but lack of space in the RBU enclosure forced us to leave it unprotected. The second strike, which was powerful enough to "fry" the turbine controller, damaged a couple of modules in a tower-mounted GBU next to the controller, but the ATLAS damage was far less severe than in the first strike. For a variety of reasons, the AOC turbine was still not back in operating status at the time the time of writing. As a result, we do not have much actual data at this time from which to draw operating experience.

Reliability of the system hardware has been a problem during the last year. We have experienced several failures in both the TTL and RS-422 communications hardware. Most of the failures appear to be the result of electrostatic discharge (ESD), not due to lightning, but due to handling in the low humidity environments found in Bushland and Albuquerque. ACRA Control has responded to these failures by identifying less susceptible components for some circuits and adding protection for others. In fact, ACRA's new Series II line, introduced late in 1999, includes many design changes to address these problems.

Our biggest concern during normal operation has been the possible loss of telemetered data from the RBU, which transmits a data frame each data acquisition cycle. This frame is merged into the master data frame which goes to the GBCU. If the RBU frame is not received, the master frame will contain the last RBU frame that it received. Since we are including data acquisition time in the data stream from the RBU, it is relatively easy to spot drop outs - we simply run a program to check the RBU acquisition times and report all data points for which the interval between acquisition times is too long.. Examination of a 20minute data file we acquired from the $A O C$ shows just 12 RBU drop outs in the 36,000 frames of data recorded. Each drop out was for a single frame - the preceding and succeeding frames were received correctly.

\section{TRANSFER OF TECHNOLOGY}

We are currently writing user's manuals for both the hardware and software, and we plan to make the ATLAS and SDAS software available for use by NREL and others in the near future. The plans, part lists, specifications, and wiring diagrams for the R.BUs and GBUs that we have built will be in the user's manual, and we will be available to assist customers with assembling ATLAS units for their jarticular application.

\section{SUMMARY}

Several enhancements have been made to the ATLAS wind turbine data acquisition hardware. Changes to the mounting of the GPS receiver provide greater flexibility on antenna mounting location, and changes to the PLD now enable the user to program the system via telemetry. Addition of a sleep mode and a $48 \mathrm{cmp}$-hour battery pack enable a rotor-based data unit to operate on battery power for up to a week between battery pack replacements.

A suite of LabVIEW-based software routines has been assembled to complement the recent advancements in data acquisition hardware. The ATLAS software allows the user to specify the ATLAS hardware configuration, the channels to be sampled, the sampling 
rate, and the setup specifics for each channel. It then programs the data acquisition units to perform the desired operations. The NREL-developed ADAS II software, enhanced to handle the continuous, long-term time-series data acquired by ATLAS, retrieves the acquired data from computer memory, displays data as directed by the user, stores data to disk, and performs a limited amount of user-directed analysis in near real time.

The time-series data files generated by ADAS II can be imported directly into several analysis routines such as the NREL-developed programs GPP, Genstats and Crunch, as well as the new SDAS program.

The SDAS software has been written to help process the large amounts of data that are typically collected in wind energy research. SDAS can retrieve a continuous stream of time-series files either from ADAS II in pseudo-real-time, or off-line from archived time-series files. It can calculate and store an extensive set of userselected and configured statistics, saving the results in a central database for later access. SDAS also has a powerful conditions-filtering capability that will allow a user to interactively interrogate the database for any set of time-series files that was previously processed by SDAS. Results can be plotted for easy viewing, displayed in Excel spreadsheets, or written to new timeseries files.

\section{REFERENCES}

1. Berg, D., Rumsey, M., Robertson, P., Kelley, N., McKenna, Ed, and Gass, K, "Development of a Light-Weight, Wind-Turbine-Rotor-Based Data Acquisition System," Paper AIAA-98-0051, Proceedings of 1998 ASME Wind Energy Symposium, Reno, NV, January 12-15, 1998, pp 238-249.

2. Berg, D. E., Robertson, P. J., and Ortiz, M. F., "Development and Application of a Light-Weight, Wind-Turbine-Rotor-Based Data Acquisition System," Windpower '98 Proceedings, Bakersfield, CA, April 27-May !, 1998.

3. Berg, D. E., and Robertson, P. J., "Precise Time Synchronization Data Acquisition with Remote Systems" Proceedings of 1998 International Telemetering Conference, San Diego, CA, October 26-29, 1998.

4. Berg, D.E., Robertson, P., and Zayas, J., "ATLAS: a Small, Light Weight, Time-Synchronized WindTurbine Data Acquisition System", AIAA 99-0050, Proceedings of 1999 ASME Wind Energy
Symposium, Reno, NV, January 11-14, 1999, pp 236-242.

5. National Instruments Corporation, 11500 North Mopac Expressway, Austin, TX 78759-3504, telephone: (512)794-0100, URL: www.ni.com.

6. ACRA Control Corporation, Landscape House, Landscape Road, Dublin 14, Ireland, telephone: +1 353-1-2951264. 